\title{
The Expanded Bead Size of Corneal C-Nerve Fibers Visualized by Corneal Confocal Microscopy Is Associated with Slow Conduction Velocity of the Peripheral Nerves in Patients with Type 2 Diabetes Mellitus
}

\author{
Fukashi Ishibashi, ${ }^{1}$ Rie Kojima, ${ }^{1}$ Miki Taniguchi, ${ }^{1}$ Aiko Kosaka, ${ }^{1}$ \\ Harumi Uetake, ${ }^{1}$ and Mitra Tavakoli ${ }^{2,3}$ \\ ${ }^{1}$ Ishibashi Clinic, 1-9-41-2 Kushido, Hatsukaichi, Hiroshima 738-0033, Japan \\ ${ }^{2}$ Centre for Endocrinology and Diabetes, Institute of Human Development, University of Manchester, 46 Grafton Street, \\ Manchester M13 9NT, UK \\ ${ }^{3}$ University of Exeter Medical School, St Luke's Campus, South Cloisters, Exeter EX1 2LU, UK \\ Correspondence should be addressed to Fukashi Ishibashi; ishiclic@urban.ne.jp and Mitra Tavakoli; m.tavakoli@exeter.ac.uk
}

Received 24 April 2016; Revised 9 June 2016; Accepted 19 June 2016

Academic Editor: Mark A. Yorek

Copyright (C) 2016 Fukashi Ishibashi et al. This is an open access article distributed under the Creative Commons Attribution License, which permits unrestricted use, distribution, and reproduction in any medium, provided the original work is properly cited.

\begin{abstract}
This study aims to establish the corneal nerve fiber (CNF) morphological alterations in a large cohort of type 2 diabetic patients and to investigate the association between the bead size, a novel parameter representing composite of accumulated mitochondria, glycogen particles, and vesicles in CNF, and the neurophysiological dysfunctions of the peripheral nerves. 162 type 2 diabetic patients and 45 healthy control subjects were studied in detail with a battery of clinical and neurological examinations and corneal confocal microscopy. Compared with controls, patients had abnormal CNF parameters. In particular the patients had reduced density and length of CNF and beading frequency and increased bead size. Alterations in CNF parameters were significant even in patients without neuropathy. The HbAlc levels were tightly associated with the bead size, which was inversely related to the motor and sensory nerve conduction velocity (NCV) and to the distal latency period of the median nerve positively. The CNF density and length positively correlated with the NCV and amplitude. The hyperglycemia-induced expansion of beads in CNF might be a predictor of slow NCV in peripheral nerves in type 2 diabetic patients.
\end{abstract}

\section{Introduction}

Diabetic neuropathy is a major complication of diabetes that may affect the sensory, autonomic, and motor nerves [1]. Mitochondria play a pivotal role in controlling nerve function by their fusion, fission, and trafficking [2, 3] and their morphological and functional anomalies play a pivotal role in developing diabetic neuropathy $[4,5]$. Corneal confocal microscopy (CCM) has been used to diagnose small C-fiber neuropathy $[6,7]$ and visualizes composites of the accumulated mitochondria, glycogen particles, and vesicles [8] as a bead [9]. In diabetic mice the presence of collections of small mitochondria and the formation of markedly enlarged mitochondria has been reported by Schmidt et al. [10]. Using IENFs of patients with diabetic neuropathy, Hamid et al. [4] observed larger mitochondrial signals composed of clustered smaller, potentially dysfunctional mitochondria or of larger predegenerative mitochondria due to a disruption in axonal transport. In small fiber neuropathy patients with relatively preserved IENFs, mitochondrial loss as judged by the significant reduction of mitochondrial oxidative phosphorylation was found [11]. However, there has been no report to associate the bead size (BS) of corneal nerve fibers (CNFs) and dysfunctions of the peripheral nerves in diabetic patients, because the size of an individual bead is too small to be assessed. We already reported that enlarging and smoothing 
of beads by S-Spline Max algorithm make it feasible to determine the size of an individual bead [12].

The aim of the present study is to associate the BS in CNFs determined by subsequent image processing with the neurophysiological dysfunctions of the peripheral nerves in patients with type 2 diabetes, elucidating whether the BS of CNFs might predict dysfunctions in the peripheral nerves.

\section{Research Design and Methods}

2.1. Subjects. 162 Japanese patients with type 2 diabetes and 45 healthy control subjects ( $\mathrm{HbAlc}<5.7 \%(38.8 \mathrm{mmol} / \mathrm{mol})$, fasting plasma glucose $<5.5 \mathrm{mmol} / \mathrm{L}$ or casual postprandial plasma glucose $<7.7 \mathrm{mmol} / \mathrm{L}$ ) were enrolled in this study at the period between May 2013 and January 2015 at the Ishibashi Clinic, Hiroshima, Japan. Subjects were excluded from the study if they consumed alcohol > one unit/day, had neurological symptoms due to neurological disorders other than diabetic neuropathy or had been diagnosed with the neuropathy due to another cause, or wore contact lenses or had history of refractive surgery or anterior segment trauma. The healthy volunteers were recruited from the general population. Written informed consent was obtained according to the Declaration of Helsinki. The Ethics Committee of the Ishibashi Clinic approved the protocol of the present research.

2.2. Laboratory Data. The HbAlc levels were converted to National Glycohemoglobin Standardization Program (NGSP) units by adding $0.4 \%$ to the measured values [13] and subsequently converted to the International Federation of Clinical Chemistry values by using the equation [(10.93NGSP) - 23.50]. The serum creatinine, lipid levels, the urinary albumin/creatinine ratio (ACR), and the estimated glomerular filtration rate (eGFR) were also determined.

\subsection{Neurophysiological Examinations. Electrophysiology} and nerve conduction velocity (NCV) studies were performed using an electromyography instrument (NeuroPak S1, NIHON KOHDEN, Tokyo, Japan). The motor conduction velocity (MCV, median nerve) and sensory conduction velocity (SCV, ulnar and sural nerve), action potential amplitudes of these three nerves, and the distal latency period of the median nerve in the wrist segment were determined.

The vibration perception threshold (VPT) was measured at the left medial malleolus using a biothesiometer (Biomedical Instruments, Newbury, OH, USA). The average of eight readings has been included. The warm and cold perception thresholds (PTs) at the dorsum of the foot were determined using a thermal stimulator that was controlled by a Peltier element (Intercross-200, Intercross Co., Tokyo, Japan). To assess the cardiovagal function of the autonomic nervous system, the coefficient of variation of R-R intervals $\left(C_{R-R}\right)$ was calculated from the R-R intervals of 200 samples on an electrocardiogram.

2.4. Assessment of Neuropathy. Severity of neuropathy and neurological deficits were assessed using the Neuropathy Disability Score (NDS) [14] which includes evaluation of vibration, pin prick, and temperature perception as well as the presence or absence of ankle reflexes to establish the severity of neuropathy: NDS $0-2$, no neuropathy; NDS 3-5, mild neuropathy; NDS 6-8, moderate neuropathy; and NDS 9-10, severe neuropathy.

2.5. CCM. All study subjects were examined using Heidelberg Retina Tomograph (HRT III, Heidelberg Engineering, Heidelberg, Germany) [9]. The technicians who were performing CCM examination were blinded to patient groups and the signs and symptoms of patients. The examined eye was anesthetized by instilling one drop of $0.4 \%$ benoxinate hydrochloride (Santen Pharmaceutical Co., Osaka, Japan). Comfort Gel (Dr. Mann Pharma, Berlin, Germany) was applied to the lens, and a disposable sterilized TomoCap (Heidelberg Engineering GmbH, Dossenheim, Germany) was mounted on the holder to cover the objective lens. After applying Comfort Gel to the TomoCap, the lens was slowly advanced until the gel touched the cornea. More than 50 images of the subbasal nerve plexus were captured using a section mode, and we analyzed at least 6 high-clarity images per subject for the quantification of the following parameters to define changes in the CNFs: (1) CNF density (CNFD): the total number of major nerve fibers $/ \mathrm{mm}^{2}$ of corneal tissue; (2) CNF length (CNFL): the total length of all nerve fibers $\left(\mathrm{mm} / \mathrm{mm}^{2}\right)$; (3) corneal nerve branch density (CNBD): the number of branches emanating from all major nerve trunks $/ \mathrm{mm}^{2}$ of corneal tissue; (4) tortuosity grade (TG); (5) frequency/0.1 mm of beading (BF); and (6) BS determined after enlarging 5 times and smoothing the original image of CCM using S-Spline Max algorithm (PhotoZoom Pro 4, Gungle Inc., Tokyo, Japan). The pixel numbers of 120 beads were counted using Photoshop Elements 8.0 (Adobe Systems Inc., San Jose, CA, USA) and averaged.

Except for the TG and BS, all measurements were performed using ImageJ (Texelcraft, Tokyo, Japan); the TG was measured by the grading system of Oliveira-Soto and Efron [15].

2.6. Statistical Analyses. The post hoc analysis of sample power revealed that, with the use of a one-sided ANOVA (significance of 0.05) and Kruskal-Wallis test, the present study population provided statistical power ranged from 0.82 to 0.99. All statistical analyses were performed using the SPSS medical package (SPSS version 19, Chicago, IL, USA). All values are presented as the mean \pm SEM. All data sets were tested for the normality using the Shapiro-Wilk test. For normally distributed variables, the comparisons between controls and total diabetic patients or subgroups stratified by NDS were made using one-way ANOVA (for continuous variables) and the $\chi^{2}$-test (for categorical variables) followed by Bonferroni corrections. For nonnormally distributed variables, the Kruskal-Wallis test was applied with subsequent Mann-Whitney $U$ test and Bonferroni corrections. The correlations between the CNF measures and the clinical factors or the results of neurophysiological tests were assessed by the Spearman rank correlation test. The sensitivity and specificity of CCM measures in differentiating between control subjects and patients without neuropathy or between patients without 
TABLE 1: Clinical characteristics and neurophysiological test of the control subjects, type 2 diabetic patients, and their subgroups stratified by the stages of neuropathy.

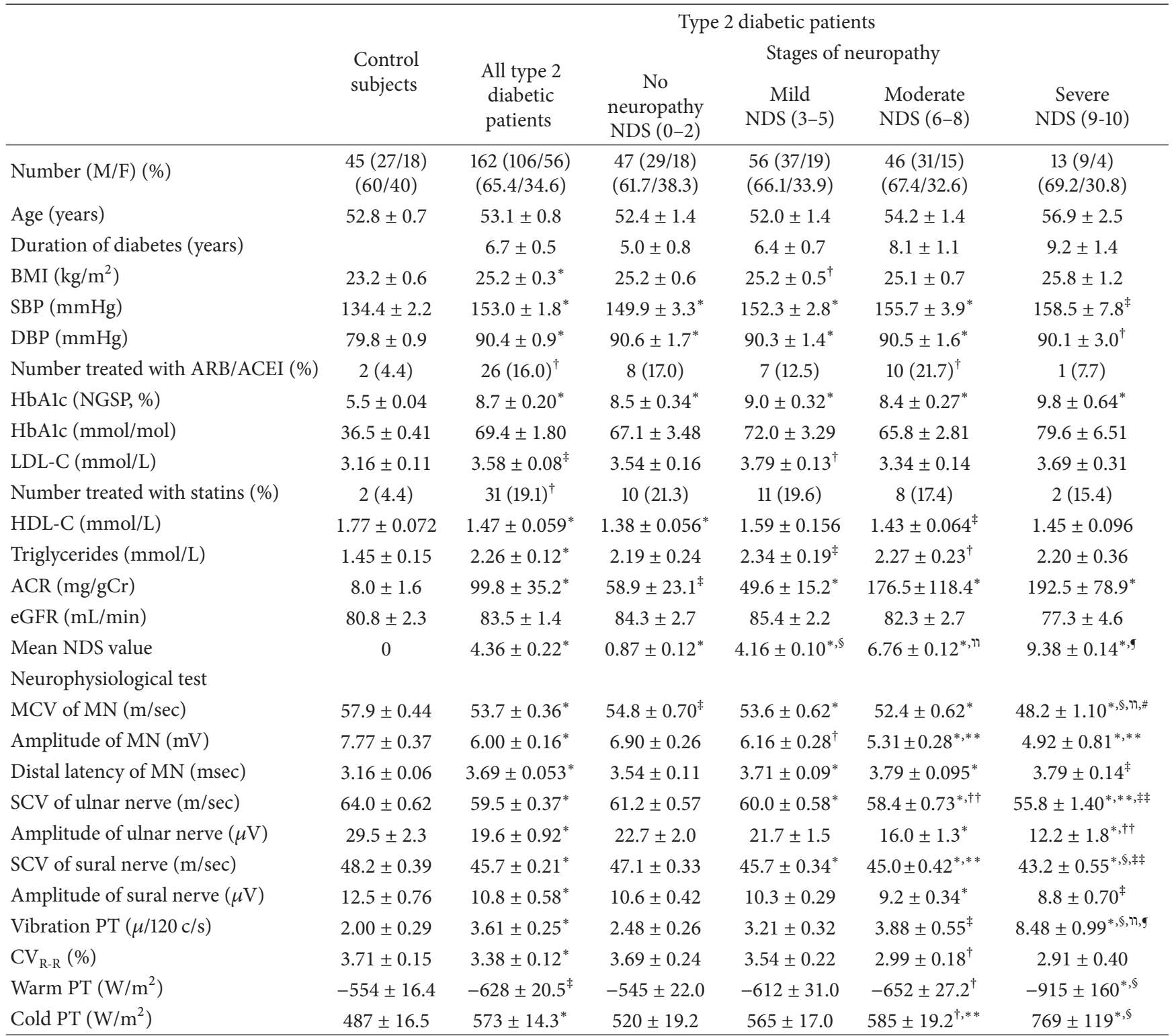

Data are the mean \pm standard error of the mean in control subjects, type 2 diabetic patients, and their subgroups stratified by the stages of the neuropathy according to the Neuropathy Disability Score (NDS) [14]. ${ }^{*} p<0.001$ compared with control subjects, ${ }^{\dagger} p<0.05$ compared with control subjects, ${ }^{\ddagger} p<0.01$ compared with control subjects, ${ }^{\S} p<0.001$ compared with patients without neuropathy, ${ }^{11} p<0.001$ compared with patients with mild neuropathy, ${ }^{9} p<0.001$ compared with patients with moderate neuropathy, ${ }^{\sharp} p<0.05$ compared with patients with moderate neuropathy, ${ }^{* *} p<0.01$ compared with patients without neuropathy, ${ }^{\dagger \dagger} p<0.05$ compared with patients without neuropathy, and ${ }^{\ddagger \ddagger} p<0.05$ compared with patients with mild neuropathy.

ACEI, angiotensin-converting enzyme inhibitor; ACR, albumin/creatinine ratio; ARB, angiotensin receptor blocker; BMI, body mass index; DBP, diastolic blood pressure; eGFR, estimated glomerular filtration rate; HDL-C, high density lipoprotein-cholesterol; LDL-C, low density lipoprotein-cholesterol; MN, median nerve; PT, perception threshold; SBP, systolic blood pressure.

neuropathy and with neuropathy were assessed using receiver operating characteristic (ROC) analysis. $p<0.05$ was considered significant.

\section{Results}

3.1. Characteristics of Healthy Controls and Patients with Type 2 Diabetes. The clinical characteristics and detailed assessment of diabetic neuropathy in diabetic patients and controls are summarized in Table 1. The gender ratio and age were similar between controls, total diabetic patients, and their subgroups. The BMI of all patients and the mild neuropathy subgroup was higher than that in controls. The blood pressure in all patients was higher than that in control subjects. The angiotensin receptor blocker or angiotensinconverting enzyme inhibitor was prescribed more frequently 
TABLE 2: Summary of corneal nerves morphological parameters in control subjects, type 2 diabetic patients, and their subgroups stratified by the stages of neuropathy.

\begin{tabular}{|c|c|c|c|c|c|c|}
\hline \multirow{3}{*}{ Corneal nerves morphological parameters } & \multirow{3}{*}{$\begin{array}{l}\text { Control } \\
\text { subjects }\end{array}$} & \multicolumn{5}{|c|}{ Patients with type 2 diabetes } \\
\hline & & \multicolumn{5}{|c|}{ Stratified by stages of neuropathy } \\
\hline & & $\begin{array}{l}\text { All type } 2 \\
\text { diabetic } \\
\text { patients }\end{array}$ & $\begin{array}{c}\text { No } \\
\text { neuropathy } \\
\text { NDS }(0-2)\end{array}$ & $\begin{array}{c}\text { Mild } \\
\text { NDS (3-5) }\end{array}$ & $\begin{array}{l}\text { Moderate } \\
\text { NDS (6-8) }\end{array}$ & $\begin{array}{c}\text { Severe } \\
\text { NDS }(9-10)\end{array}$ \\
\hline Corneal nerve fiber density (CNFD) $\left(\mathrm{no} / \mathrm{mm}^{2}\right)$ & $33.0 \pm 1.2$ & $23.3 \pm 0.41^{*}$ & $25.3 \pm 0.72^{*}$ & $23.5 \pm 0.66^{*}$ & $21.7 \pm 0.83^{*, \dagger}$ & $21.6 \pm 1.13^{*}$ \\
\hline Corneal nerve fiber length (CNFL) $\left(\mathrm{mm} / \mathrm{mm}^{2}\right)$ & $15.5 \pm 0.53$ & $9.5 \pm 0.16^{*}$ & $12.3 \pm 0.31^{*}$ & $11.7 \pm 0.30^{*}$ & $11.0 \pm 0.38^{*, \dagger}$ & $11.3 \pm 0.49^{*}$ \\
\hline Corneal nerve branch density (CNBD) $\left(\mathrm{no} / \mathrm{mm}^{2}\right)$ & $14.5 \pm 1.2$ & $9.6 \pm 0.26^{*}$ & $9.5 \pm 0.39^{\ddagger}$ & $10.0 \pm 0.51^{\ddagger}$ & $9.3 \pm 0.54^{\ddagger}$ & $9.1 \pm 0.67^{\S}$ \\
\hline Tortuosity grade (TG) & $1.98 \pm 0.06$ & $2.49 \pm 0.03^{*}$ & $2.47 \pm 0.05^{*}$ & $2.51 \pm 0.05^{*}$ & $2.47 \pm 0.05^{*}$ & $2.54 \pm 0.13^{*}$ \\
\hline Beading frequency $(\mathrm{BF})$ (number/0.1 mm) & $23.7 \pm 0.29$ & $20.4 \pm 0.16^{*}$ & $20.1 \pm 0.27^{*}$ & $20.9 \pm 0.32^{*}$ & $20.3 \pm 0.27^{*}$ & $19.6 \pm 0.36^{*}$ \\
\hline Bead size $(\mathrm{BS})\left(\mu \mathrm{m}^{2}\right)$ & $7.94 \pm 0.07$ & $9.84 \pm 0.05^{*}$ & $9.65 \pm 0.09^{*}$ & $9.82 \pm 0.08^{*}$ & $9.95 \pm 0.09^{*}$ & $10.3 \pm 0.17^{*, \dagger}$ \\
\hline
\end{tabular}

Data are expressed as mean \pm standard error of the mean (SEM) in control subjects, type 2 diabetic patients, and their subgroups stratified by the Neuropathy Disability Score (NDS). ${ }^{*} p<0.001$ compared with control subjects, ${ }^{\dagger} p<0.05$ compared with patients without neuropathy, ${ }^{\ddagger} p<0.01$ compared with control subjects, and ${ }^{\S} p<0.05$ compared with control subjects.

for the total patient group and the moderate neuropathy subgroup than for controls. The HbAlc levels in the total patient group and all diabetic subgroups were higher than those in controls, while no difference was found between diabetic subgroups. The LDL-cholesterol level in the total patient group and a mild neuropathy subgroup was elevated compared with that in controls. Statins were prescribed more frequently for the total diabetic patients than for the controls. The HDL-cholesterol level in the total patient group and patients without neuropathy and with moderate neuropathy was lower than that in controls. The triglycerides level in the total patient group and subgroups with mild or moderate neuropathy was increased compared with that in controls. The ACR in the whole patient group and all diabetic subgroups was significantly higher than controls. There were significant differences in the mean NDS between all subgroups of diabetic patients stratified by NDS.

3.2. Neurological Examinations. Except for the MCV of the median nerve, there existed no difference in the neurophysiological tests between controls and the subgroup without neuropathy. The MCV, amplitude, and distal latency period of the median nerve and the SCV and amplitude of ulnar and sural nerve deteriorated relative to the severity of neuropathy (Table 1). VPT increased relative to the severity of neuropathy. $\mathrm{CV}_{\mathrm{R}-\mathrm{R}}$ and temperature PTs in the moderate neuropathy subgroup were impaired compared with those in controls or patients without neuropathy (Table 1).

\subsection{Corneal Nerves Morphological Parameters in Control} Subjects and Type 2 Diabetic Patients. In the cohort of patients without neuropathy, the CNFD, CNFL, CNBD, and BF were markedly reduced, and TG and BS were increased compared with controls. The further mild deterioration of CNFD and CNFL was found in the moderate neuropathy subgroup and BS in patients with severe neuropathy (Table 2). BS in patients without neuropathy expanded compared with that of controls and further expansion was found only in patients with severe neuropathy (Figure 1).

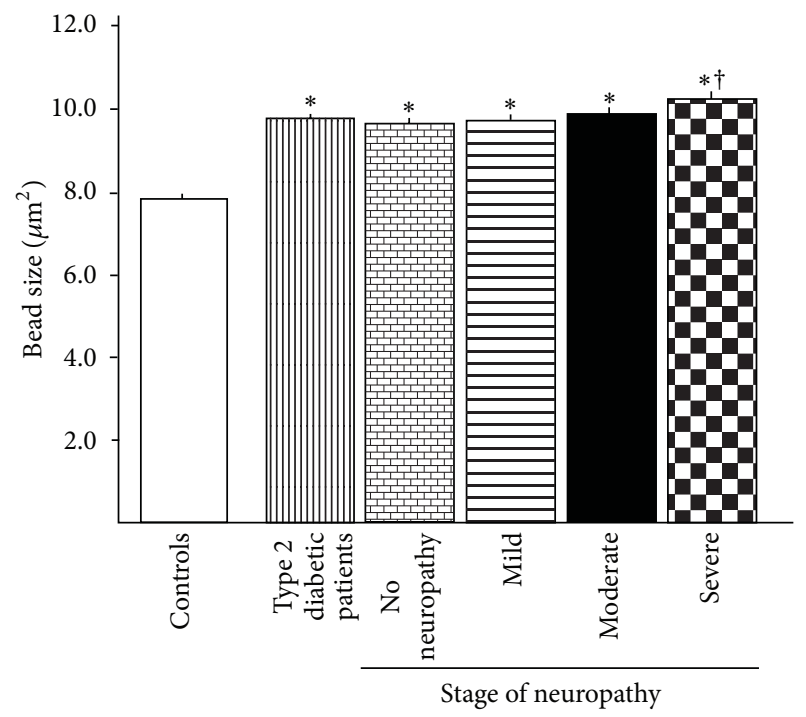

FIGURE 1: Comparison of the bead sizes of the corneal nerve fibers between control subjects, total type 2 diabetic patients, and diabetic subgroups stratified by the severity of neuropathy. Data are the mean \pm SEM. ${ }^{*} p<0.001$ compared with control subjects and ${ }^{\dagger} p<0.05$ compared with patients without neuropathy.

Figure 2 illustrated the representative beads in original CCM images (a), when the images were simply magnified 5 times by Photoshop (b), and when the images were enlarged 5 times with smoothing by the S-Spline Max algorithm (c) in a control subject (1), a patient without diabetic neuropathy (2), a patient with mild diabetic neuropathy (3), a patient with moderate diabetic neuropathy (4), or a patient with severe diabetic neuropathy (5). The images that were simply magnified by Photoshop were not suitable to determine the pixel numbers accurately. In comparison, the images processed by the S-Spline Max algorithm were clearly demarcated, and the pixel numbers were easily determined. Compared with the BS of controls, those in diabetic patients appeared to expand. The intrarater variability (averaged CV) in the measurement 


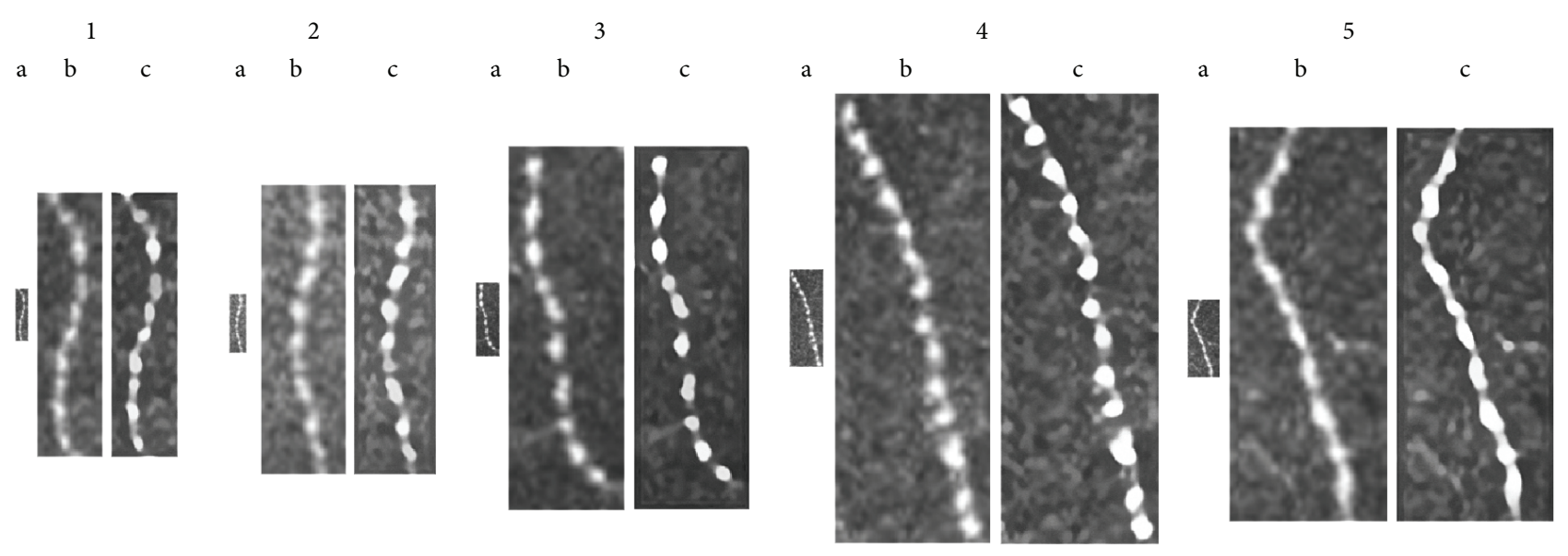

FIGURE 2: The representative beads in the original CCM images (a), images simply magnified 5 times by Photoshop (b), and images enlarged 5 times with smoothing by the S-Spline Max algorithm (c) in a control subject (1, male, 56 years, NDS: 0), patient without neuropathy (2, male, 52 years, NDS: 1), patient with mild neuropathy (3, male, 54 years, NDS: 5 ), patient with moderate neuropathy (4, male, 56 years, NDS: $7)$, and patient with severe neuropathy (5, male, 57 years, NDS: 9).

TABLE 3: Differentiating efficacy of corneal nerve fiber parameters as AUC and $p$ values with CCM cut-off with sensitivity and specificity between control subjects and type 2 diabetic patients without neuropathy or between type 2 diabetic patients with or without neuropathy.

\begin{tabular}{|c|c|c|c|c|c|c|c|c|c|c|}
\hline \multirow[t]{2}{*}{ Variable } & \multicolumn{5}{|c|}{$\begin{array}{l}\text { Between control subjects and type } 2 \text { diabetic } \\
\text { patients without neuropathy }\end{array}$} & \multicolumn{5}{|c|}{$\begin{array}{c}\text { Between type } 2 \text { diabetic patients without or } \\
\text { with neuropathy }\end{array}$} \\
\hline & AUC & $p$ value & Cut-off & Sensitivity & Specificity & AUC & $p$ value & Cut-off & Sensitivity & Specificity \\
\hline CNFD & 0.847 & $<0.0001$ & $28.1 / \mathrm{mm}^{2}$ & 0.79 & 0.78 & 0.610 & 0.020 & $23.1 / \mathrm{mm}^{2}$ & 0.66 & 0.54 \\
\hline CNFL & 0.830 & $<0.0001$ & $13.4 \mathrm{~mm} / \mathrm{mm}^{2}$ & 0.79 & 0.68 & 0.585 & 0.071 & $11.6 \mathrm{~mm} / \mathrm{mm}^{2}$ & 0.63 & 0.55 \\
\hline $\mathrm{BF}$ & 0.883 & $<0.0001$ & $22.1 / 0.1 \mathrm{~mm}$ & 0.92 & 0.83 & 0.538 & 0.427 & $20.5 / 0.1 \mathrm{~mm}$ & 0.48 & 0.66 \\
\hline BS & 0.997 & $<0.0001$ & $8.47 \mu \mathrm{m}^{2}$ & 0.99 & 0.96 & 0.602 & 0.031 & $9.76 \mu \mathrm{m}^{2}$ & 0.65 & 0.53 \\
\hline
\end{tabular}

$\mathrm{BF}$, beading frequency; BS, bead size; CNFD, corneal nerve fiber density; CNFL, corneal nerve fiber length.

of the pixel numbers of bead in 10 control subjects was $12.7 \pm$ $2.6 \%$.

According to the ROC curves for the four CCM parameters between controls and patients without neuropathy (NDS < 3) (Figure 3 and Table 3), AUC was 0.847 for CNFD, 0.830 for CNFL, 0.883 for BF, and 0.997 for BS. Because the AUC, sensitivity, and specificity of BS are the best among parameters of CCM, it is considered that BS is the most reliable marker of $\mathrm{CNF}$ alteration in type 2 diabetes before developing neuropathy (Table 3). On the other hand, AUC between patients without neuropathy (NDS < 3) and with neuropathy (NDS $>3$ ) did not seem to be useful in detecting the presence of neuropathy: AUC was 0.610 for CNFD, 0.585 for CNFL, 0.538 for BF, and 0.602 for BS (Table 3).

\subsection{Correlations between CCM Parameters and Clinical Fac-} tors or Neurophysiological Tests. There was significant correlation between HbAlc and all CNF parameters except TG and BF. NDS was associated with CNFD and CNFL inversely and with BS positively (Table 4). The BS had the robust inverse association with MCV and the positive association with the distal latency period of the median nerve and inversely related with the SCV of the ulnar and sural nerve. On the other hand, CNFD and CNFL had the modest association with the NCV as well as the amplitudes of the peripheral nerves. The weak but significant relationships between the CNFD, CNFL, and $\mathrm{CNBD}$ and $\mathrm{CV}_{\mathrm{R}-\mathrm{R}}$ or cold PT were found, while BS had the positive relationship with a VPT (Table 4).

\section{Discussion}

Diabetic neuropathy is a major complication of diabetes and leads to significant morbidity and mortality [16]. The chronic hyperglycemia-induced changes in mitochondrial structure, function, and dynamics may lead to the development of diabetic neuropathy $[2,3,17]$. The increase in the number of small mitochondria due to excessive fission in the dorsal root fibers of $\mathrm{db} / \mathrm{db}$ mouse with diabetic neuropathy has been reported [18]. In a study with Akita diabetic mouse with diabetic neuropathy, sympathetic ganglia show the accumulation of minute mitochondria [19]. In patients with diabetic neuropathy the larger mitochondrial signals composed of clustered smaller, potentially dysfunctional mitochondria or of larger predegenerative mitochondria in IENFs due to a disruption in axonal transport were observed [4]. In the small fiber neuropathy patients in early stage, mitochondrial loss was reported [11]. Since an invasive skin or nerve biopsy is mandatory for the morphological assessment of mitochondrial number and size, it could not be performed as a clinical routine procedure. 

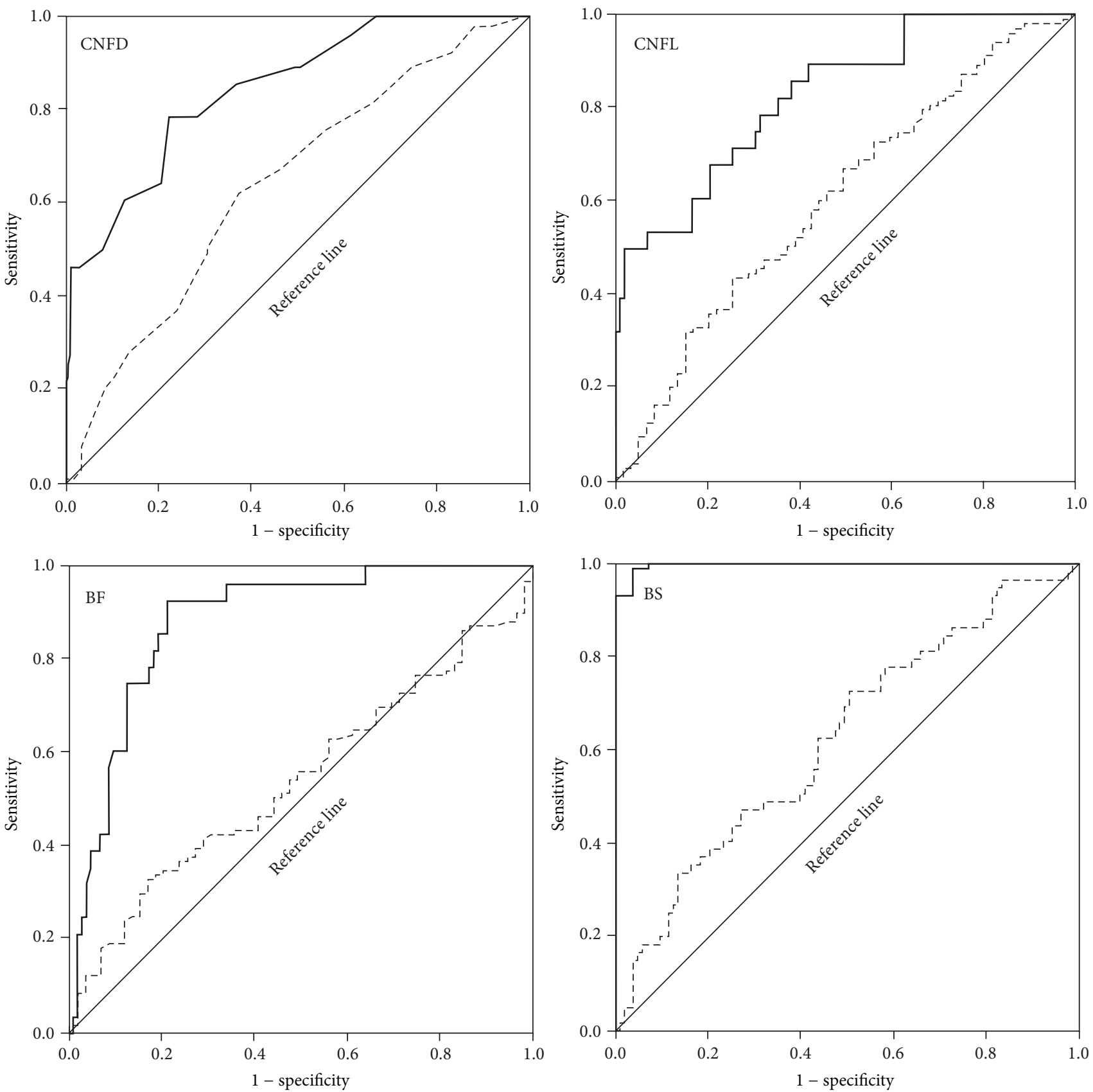

FIGURE 3: Receiver operating characteristic (ROC) curve analyses for CNFD, CNFL, BF, and BS between control subjects and patients without neuropathy (NDS < 3) (-) and between patients without neuropathy (NDS < 3) and with neuropathy (NDS > 3) $(---)$.

To our knowledge, this is the largest cohort of type 2 diabetic patients that have been studied in detail for standard clinical examinations and CCM, and the new parameters including BS have been studied for the first time.

The CNF pathology assessed by CCM has been proposed as a surrogate marker for a small fiber neuropathy in patients with diabetes $[6,7]$, because the ROC curves for the CCM parameters (CNFD, CNFL, and CNBD) for NDS $>3$ revealed good AUC, sensitivity, and specificity [6] and because the CCM analysis using a conventional and novel algorithm to reconstruct CNF images had revealed the significant correlations between CNFD, CNFL, or CNBD and the NCV, VPT, temperature PT, heart rate variability, or the clinical severity of diabetic neuropathy [20]. The ROC analysis of CCM parameters in the present study revealed that the AUC, sensitivity, and specificity of CCM measures in differentiating between control subjects and patients without neuropathy (NDS < 3) were excellent and among the parameters of CNF morphology, BS had the best AUC, sensitivity, and specificity.

Using the thin section of a freshly isolated human cornea the electron microscopy can identify beads in CNFs as a composite of the accumulated mitochondria, glycogen 
TABLE 4: Relationship between the various measures of corneal nerve fibers and clinical and neurophysiological parameters in patients with type 2 diabetes.

\begin{tabular}{|c|c|c|c|c|c|c|c|c|c|c|c|c|}
\hline & \multicolumn{2}{|c|}{ CNFD } & \multicolumn{2}{|c|}{ CNFL } & \multicolumn{2}{|c|}{ CNBD } & \multicolumn{2}{|c|}{ TG } & \multicolumn{2}{|c|}{$\mathrm{BF}$} & \multicolumn{2}{|c|}{ Bead size } \\
\hline & $\beta$ & $p$ & $\beta$ & $p$ & $\beta$ & $p$ & $\beta$ & $p$ & $\beta$ & $p$ & $\beta$ & $p$ \\
\hline Gender & -0.056 & 0.482 & -0.054 & 0.498 & 0.093 & 0.240 & -0.040 & 0.617 & -0.083 & 0.295 & 0.034 & 0.669 \\
\hline Age & -0.012 & 0.882 & -0.060 & 0.450 & -0.066 & 0.405 & 0.004 & 0.959 & -0.026 & 0.744 & 0.057 & 0.475 \\
\hline Duration of DM & -0.094 & 0.236 & -0.083 & 0.291 & -0.071 & 0.372 & 0.036 & 0.645 & -0.025 & 0.750 & -0.040 & 0.617 \\
\hline SBP & -0.107 & 0.176 & -0.105 & 0.183 & 0.011 & 0.886 & -0.107 & 0.174 & -0.010 & 0.902 & -0.032 & 0.685 \\
\hline DBP & -0.132 & 0.093 & -0.113 & 0.150 & 0.015 & 0.848 & -0.139 & 0.079 & -0.004 & 0.961 & 0.017 & 0.830 \\
\hline HbAlc & -0.200 & 0.011 & -0.213 & 0.007 & -0.168 & 0.032 & 0.003 & 0.973 & -0.080 & 0.313 & 0.352 & $<0.0001$ \\
\hline LDL-C & 0.028 & 0.724 & 0.011 & 0.891 & -0.007 & 0.925 & 0.075 & 0.343 & -0.116 & 0.141 & -0.006 & 0.937 \\
\hline HDL-C & -0.052 & 0.509 & -0.105 & 0.182 & -0.066 & & 0.032 & 0.683 & 0.027 & & -0.043 & 0.586 \\
\hline Triglycerides & -0.038 & 0.633 & -0.060 & 0.451 & -0.077 & 0.330 & 0.034 & & -0.015 & & 0.002 & 0.984 \\
\hline NDS & -0.255 & 0.001 & -0.208 & 0.008 & -0.019 & 0.815 & 0.034 & & -0.012 & 0.883 & 0.203 & 0.010 \\
\hline MCV of MN & 0.199 & 0.011 & 0.193 & 0.014 & 0.054 & 0.494 & 0.063 & 0.426 & 0.050 & 0.526 & -0.358 & $<0.0001$ \\
\hline Amplitude of MN & 0.208 & 0.008 & 0.172 & 0.028 & -0.009 & 0.914 & -0.083 & 0.292 & -0.028 & 0.722 & 0.091 & 0.248 \\
\hline Distal latency of MN & -0.090 & 0.256 & -0.090 & 0.256 & -0.018 & 0.819 & -0.083 & 0.295 & 0.065 & 0.413 & 0.294 & $<0.0001$ \\
\hline SCV of UN & 0.200 & 0.011 & 0.225 & 0.004 & 0.138 & 0.079 & 0.070 & 0.379 & 0.037 & 0.644 & -0.273 & $<0.0001$ \\
\hline Amplitude of UN & 0.195 & 0.013 & 0.215 & 0.006 & 0.040 & 0.616 & -0.031 & 0.699 & 0.053 & 0.504 & 0.083 & 0.294 \\
\hline SCV of SN & 0.201 & 0.010 & 0.223 & 0.004 & 0.107 & 0.175 & 0.105 & 0.183 & 0.031 & 0.698 & -0.237 & 0.002 \\
\hline Amplitude of SN & 0.224 & 0.004 & 0.232 & 0.003 & 0.076 & 0.339 & -0.064 & 0.417 & 0.098 & 0.215 & 0.004 & 0.963 \\
\hline Vibration PT & -0.019 & 0.812 & 0.029 & 0.803 & 0.016 & 0.837 & 0.097 & 0.220 & -0.046 & 0.562 & 0.199 & 0.011 \\
\hline $\mathrm{CV}_{\mathrm{R}-\mathrm{R}}$ & 0.161 & 0.040 & 0.210 & 0.007 & 0.170 & 0.030 & -0.026 & 0.744 & 0.009 & 0.909 & -0.098 & 0.216 \\
\hline Warm PT & 0.021 & 0.790 & -0.002 & 0.985 & -0.073 & 0.358 & -0.149 & 0.058 & -0.025 & 0.753 & 0.051 & 0.523 \\
\hline Cold PT & -0.142 & 0.071 & -0.157 & 0.046 & -0.015 & 0.853 & -0.030 & 0.706 & -0.028 & 0.727 & 0.025 & 0.754 \\
\hline
\end{tabular}

$\mathrm{BF}$, beading frequency; $\mathrm{CNBD}$, corneal nerve branch density; CNFD, corneal nerve fiber density; CNFL, corneal nerve fiber length; CV, coefficient of variation; DBP, diastolic blood pressure; DM, diabetes mellitus; HDL-C, high density lipoprotein-cholesterol; LDL-C, low density lipoprotein-cholesterol; MCV, motor conduction velocity; MN, median nerve; NDS, Neuropathy Disability Score; PT, perception threshold; SBP, systolic blood pressure; SCV, sensory conduction velocity; SN, sural nerve; TG, tortuosity grade; UN, ulnar nerve.

particles, and vesicles $[8,21]$ playing some role in controlling CNF functions [21] with similar ultrastructure to nociceptor terminals. The present study revealed that BF decreased and BS expanded in patients with diabetes compared with control subjects. In the present study BS inversely associated with CNFD $(r=-0.269, p<0.001)$, CNFL $(r=-0.212, p=$ $0.003)$, and CNBD $(r=-0.229, p=0.001)$, and BF had positive relationship with CNFD $(r=0.252, p<0.001)$, CNFL $(r=0.205, p=0.005)$, and CNBD $(r=0.184, p=$ 0.011 ). Therefore, altered beading structures may have relationship with the loss of main nerve fibers as well as branches. Although the increased irregularity in the periodicity of CNF beading has been reported in streptozotocin- (STZ-) diabetic rat [22], alteration in the BS and in composition of bead remained to be clarified. The axonal trafficking of mitochondria is controlled by cytoskeletons, motor proteins, and ATP fuel supply [23]. In STZ-diabetic rats, axonal neurofilaments in peripheral nerve were deleted [24], and sciatic level of kinesin $5 \mathrm{~B}$ motor protein which is involved in the axonal transport of mitochondria is changed [25]. These results suggested that changes in the distribution of mitochondria in CNFs might occur resulting in altered number and size of beads. However, we could not determine the exact mitochondrial area in bead using mitochondria marker, because the present study was an in vivo human study using CCM.
Therefore, the contribution of mitochondrial number and size to the BS remained to be determined. In patients with diabetic neuropathy [26] and the spontaneously diabetic BBWistar-rat [27], mitochondrial accumulation of glycogen particles was observed. In experimental diabetic animals, the loss of vesicles in presynapse [28] and autonomic nerve endings [29] was reported. Therefore, the alterations in components of bead other than mitochondria might influence the density and size of bead.

The present research found the significant relationships between parameters of main CNF and branch and the NCV and amplitudes of the peripheral nerves, heart rate variability, and a cold PT. Although we and others previously reported the preliminary findings of lower BF of CNFs in diabetic patients [30-32], the relationship between the BS and neurophysiological tests had never been investigated. The beads can be clearly demonstrated along CNFs by CCM. However, the individual bead in the original CCM image is too small for assessing its area. The enlarging and smoothing of the original CCM image by S-Spline Max algorithm enable us to determine the size of an individual bead in CNFs in spite of the uncertain accuracy and exactness of this method.

Based on the current results, CCM can detect CNF alteration in patients without clinical evidence of neuropathy compared with those of the controls. According to ROC 
analysis BS had the largest AUC with the best sensitivity and specificity compared with CNFD, CNFL, and BF. These results indicated that the diabetes-induced expansion of BS and changes in other morphological parameters of CCM might have predictive value for the dysfunctions of the peripheral nerves. The expansion of beads occurred in patients without the neuropathy, getting larger in patients with severe neuropathy. The BS had good negative relationship with MCV and SCV of the peripheral nerves and directly associated with a distal latency period of the median nerve. In the present study the BS correlated strongly to HbAlc levels. In myelinated murine axon, the oxidative stress alters the external morphology and reduces the transport of mitochondria at the nodes of Ranvier. These mitochondrial changes expand from the node of Ranvier bidirectionally [33]. In STZdiabetic mice, there is a significant increase in synaptic delay compared with control mice, and the accumulation of degenerated mitochondria of presynaptic axon of the neuromuscular junction was observed [34]. However, because there has been no report investigating the morphological changes in mitochondria of CNFs in patients with diabetes, we could not assume that the bead in the CNFs is a surrogate marker of the size of mitochondrial area in the peripheral nerves in diabetic patients.

We acknowledge limitations to the present study, which may affect the interpretation of the results. First, although we measured the BS of CNF in patients with type 2 diabetes, we did not determine whether the number of mitochondria in an expanded bead in CNFs increased or not. Furthermore, alteration in other components of bead could influence BS. Second, we assessed BS after enlarging and smoothing of original bead. However, the accuracy and preciseness of this method were not established. The future improvement in a resolution of CCM apparatus will make it possible to assess the BS directly using an original CCM image. Lastly, the potential bias determining BF and BS by human errors could not be ruled out. The automatic analyzing system for beading as already developed for other corneal morphological parameters of CNFs [35] would eliminate this type of errors.

\section{Conclusions}

In conclusion, the expansion of beads of the CNFs in patients with type 2 diabetes occurred before the development of neuropathy and was related to the slow NCV and the prolonged distal latency period of the peripheral nerves. The BS in the CNFs has a predictive value for developing the slow NCV of the peripheral nerves in patients with type 2 diabetes. However, elucidating the mechanisms of the expansion of bead in CNFs was beyond the scope of the present study.

\section{Disclosure}

Fukashi Ishibashi and Mitra Tavakoli are the guarantors of this work and as such had full access to all data in the study and take responsibility for the integrity of the data and the accuracy of the data analysis and interpretation.

\section{Competing Interests}

This study received no financial support. The authors have reported no competing interests.

\section{Authors' Contributions}

Fukashi Ishibashi designed the study, researched data, and wrote the entire paper. Rie Kojima and Miki Taniguchi performed a CCM examination and neurophysiological tests. Aiko Kosaka and Harumi Uetake gathered the clinical and laboratory data and statistically analyzed all data. Mitra Tavakoli advised on the statistical analysis, interpreted the results, and reviewed and revised the whole paper.

\section{References}

[1] P. J. Dyck and C. Giannini, "Pathologic alterations in the diabetic neuropathies of humans: a review," Journal of Neuropathology and Experimental Neurology, vol. 55, no. 12, pp. 1181-1193, 1996.

[2] R. H. Baloh, "Mitochondrial dynamics and peripheral neuropathy," Neuroscientist, vol. 14, no. 1, pp. 12-18, 2008.

[3] S. Frank, "Dysregulation of mitochondrial fusion and fission: an emerging concept in neurodegeneration," Acta Neuropathologica, vol. 111, no. 2, pp. 93-100, 2006.

[4] H. S. Hamid, C. M. Mervak, and A. E. Münch, "Hyperglycemiaand neuropathy-induced changes in mitochondria within sensory nerves," Annals of Clinical and Translational Neurology, vol. 1, no. 10, pp. 799-812, 2014.

[5] J. L. Edwards, A. Quattrini, S. I. Lentz et al., "Diabetes regulates mitochondrial biogenesis and fission in mouse neurons," Diabetologia, vol. 53, no. 1, pp. 160-169, 2010.

[6] M. Tavakoli, C. Quattrini, C. Abbott et al., "Corneal confocal microscopy: a novel noninvasive test to diagnose and stratify the severity of human diabetic neuropathy," Diabetes Care, vol. 33, no. 8, pp. 1792-1797, 2010.

[7] D. Ziegler, N. Papanas, A. Zhivov et al., "Early detection of nerve fiber loss by corneal confocal microscopy and skin biopsy in recently diagnosed type 2 diabetes," Diabetes, vol. 63, no. 7, pp. 2454-2463, 2014.

[8] L. J. Müller, L. Pels, and G. F. J. M. Vrensen, "Ultrastructural organization of human corneal nerves," Investigative Ophthalmology and Visual Science, vol. 37, no. 4, pp. 476-488, 1996.

[9] F. Ishibashi, M. Okino, M. Ishibashi et al., "Corneal nerve fiber pathology in Japanese type 1 diabetic patients and its correlation with antecedent glycemic control and blood pressure," Journal of Diabetes Investigation, vol. 3, no. 2, pp. 191-198, 2012.

[10] R. E. Schmidt, C. A. Parvin, and K. G. Green, "Synaptic ultrastructural alterations anticipate the development of neuroaxonal dystrophy in sympathetic ganglia of aged and diabetic mice," Journal of Neuropathology and Experimental Neurology, vol. 67, no. 12, pp. 1166-1186, 2008.

[11] J. Casanova-Molla, M. Morales, G. Garrabou et al., "Mitochondrial loss indicates early axonal damage in small fiber neuropathies," Journal of the Peripheral Nervous System, vol. 17, no. 2, pp. 147-157, 2012.

[12] F. Ishibashi, "Glycemic state has real-time impact on beading size and frequency while requiring several years to influence nerve fibers by corneal confocal microscopy," Diabetes, vol. 61, supplement 1, p. A148, 2012. 
[13] A. Kashiwagi, M. Kasuga, E. Araki et al., "International clinical harmonization of glycated hemoglobin in Japan: from Japan Diabetes Society to National Glycohemoglobin Standardization Program values," Journal of Diabetes Investigation, vol. 3, no. 1, pp. 39-40, 2012.

[14] M. J. Young, A. J. M. Boulton, A. F. Macleod, D. R. R. Williams, and P. H. Sonksen, "A multicentre study of the prevalence of diabetic peripheral neuropathy in the United Kingdom hospital clinic population," Diabetologia, vol. 36, no. 2, pp. 150-154, 1993.

[15] L. Oliveira-Soto and N. Efron, "Morphology of corneal nerves using confocal microscopy," Cornea, vol. 20, no. 4, pp. 374-384, 2001.

[16] A. I. Vinik, T. S. Park, K. B. Stansberry, and G. L. Pittenger, “Diabetic neuropathies," Diabetologia, vol. 43, no. 8, pp. 957973, 2000.

[17] N. Hernández-Beltrán, C. B. Moreno, and A. M. GutiérrezÁlvarez, "Contribution of mitochondria to pain in diabetic neuropathy," Endocrinología y Nutrición, vol. 60, no. 1, pp. 2532, 2013.

[18] A. M. Vincent, J. L. Edwards, L. L. McLean et al., "Mitochondrial biogenesis and fission in axons in cell culture and animal models of diabetic neuropathy," Acta Neuropathologica, vol. 120, no. 4, pp. 477-489, 2010.

[19] R. E. Schmidt, K. G. Green, L. L. Snipes, and D. Feng, "Neuritic dystrophy and neuronopathy in Akita (Ins2 ${ }^{\text {Akita }}$ ) diabetic mouse sympathetic ganglia," Experimental Neurology, vol. 216, no. 1, pp. 207-218, 2009.

[20] N. Papanas and D. Ziegler, "Corneal confocal microscopy: recent progress in the evaluation of diabetic neuropathy," Journal of Diabetes Investigation, vol. 6, no. 4, pp. 381-389, 2015.

[21] L. J. Müller, C. F. Marfurt, F. Kruse, and T. M. T. Tervo, "Corneal nerves: structure, contents and function," Experimental Eye Research, vol. 76, no. 5, pp. 521-542, 2003.

[22] N. Ishida, G. N. Rao, M. Del Cerro, and J. V. Aquavella, "Corneal nerve alterations in diabetes mellitus," Archives of Ophthalmology, vol. 102, no. 9, pp. 1380-1384, 1984.

[23] K. J. De Vos, A. J. Grierson, S. Ackerley, and C. C. J. Miller, "Role of axonal transport in neurodegenerative diseases," Annual Review of Neuroscience, vol. 31, pp. 151-173, 2008.

[24] S. Yagihashi, M. Kamijo, and K. Watanabe, "Reduced myelinated fiber size correlates with loss of axonal neurofilaments in peripheral nerve of chronically streptozotocin diabetic rats," The American Journal of Pathology, vol.136, no. 6, pp. 1365-1373, 1990.

[25] M. Rahmati, R. Gharakhanlou, M. Movahedin et al., "Treadmill training modifies KIF5B motor protein in the STZ-induced diabetic rat spinal cord and sciatic nerve," Archives of Iranian Medicine, vol. 18, no. 2, pp. 94-101, 2015.

[26] S. Yagihashi and M. Matsunaga, "Ultrastructural pathology of peripheral nerves in patients with diabetic neuropathy," Tohoku Journal of Experimental Medicine, vol. 129, no. 4, pp. 357-366, 1979.

[27] A. A. F. Sima, "Peripheral neuropathy in the spontaneously diabetic BB-Wistar-rat. An ultrastructural study," Acta Neuropathologica, vol. 51, no. 3, pp. 223-227, 1980.

[28] J. S. Ahn, T. H. Lee, and M. C. Lee, "Ultrastructure of neuromuscular junction in vacor-induced diabetic rats," The Korean Journal of Internal Medicine, vol. 13, no. 1, pp. 47-50, 1998.

[29] S. N. Sanyal, T. Wada, M. Yamabe et al., "Synaptic degradation of cardiac autonomic nerves in streptozotocin-induced diabetic rats," Pathophysiology, vol. 19, no. 4, pp. 299-307, 2012.
[30] F. Ishibashi, A. Kawasaki, E. Yamanaka, A. Kosaka, and H. Uetake, "Morphometric features of corneal epithelial basal cells, and their relationship with corneal nerve pathology and clinical factors in patients with type 2 diabetes," Journal of Diabetes Investigation, vol. 4, no. 5, pp. 492-501, 2013.

[31] F. Ishibashi, R. Kojima, A. Kawasaki, E. Yamanaka, A. Kosaka, and H. Uetake, "Correlation between sudomotor function, sweat gland duct size and corneal nerve fiber pathology in patients with type 2 diabetes mellitus," Journal of Diabetes Investigation, vol. 5, no. 5, pp. 588-596, 2014.

[32] E. Maddaloni, F. Sabatino, R. Del Toro et al., "In vivo corneal confocal microscopy as a novel non-invasive tool to investigate cardiac autonomic neuropathy in type 1 diabetes," Diabetic Medicine, vol. 32, no. 2, pp. 262-266, 2015.

[33] H. Bros, J. M. Millward, F. Paul, R. Niesner, and C. InfanteDuarte, "Oxidative damage to mitochondria at the nodes of Ranvier precedes axon degeneration in ex vivo transected axons," Experimental Neurology, vol. 261, pp. 127-135, 2014.

[34] M. A. Fahim, F. El-Sabban, and N. Davidson, "Muscle contractility decrement and correlated morphology during the pathogenesis of streptozotocin-diabetic mice," Anatomical Record, vol. 251, no. 2, pp. 240-244, 1998.

[35] M. A. Dabbah, J. Graham, I. N. Petropoulos, M. Tavakoli, and R. A. Malik, "Automatic analysis of diabetic peripheral neuropathy using multi-scale quantitative morphology of nerve fibres in corneal confocal microscopy imaging," Medical Image Analysis, vol. 15, no. 5, pp. 738-747, 2011. 


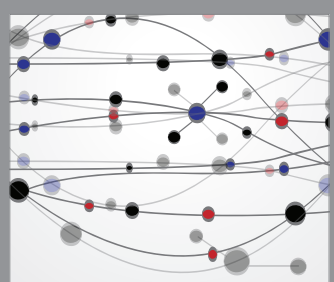

The Scientific World Journal
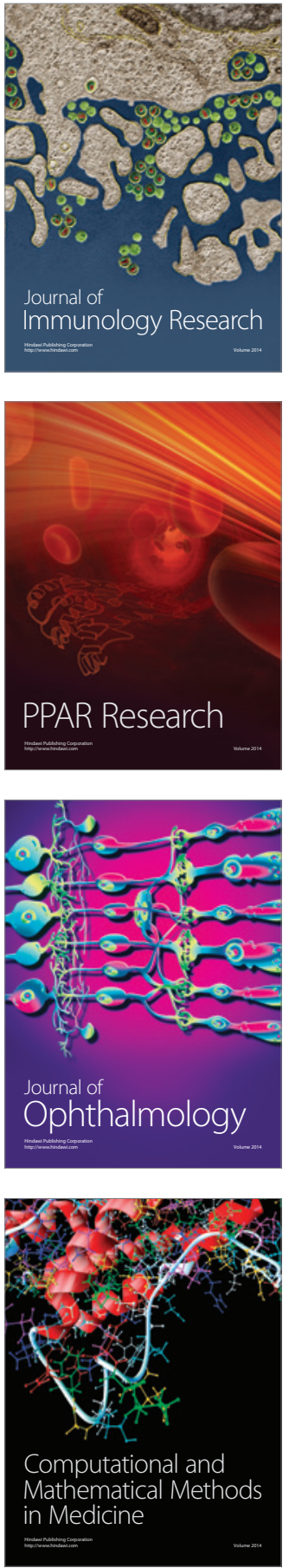

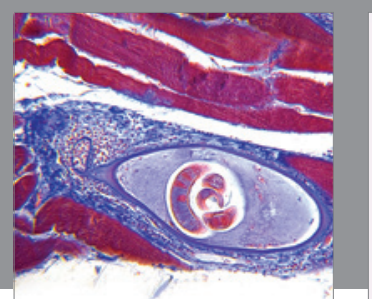

Gastroenterology Research and Practice

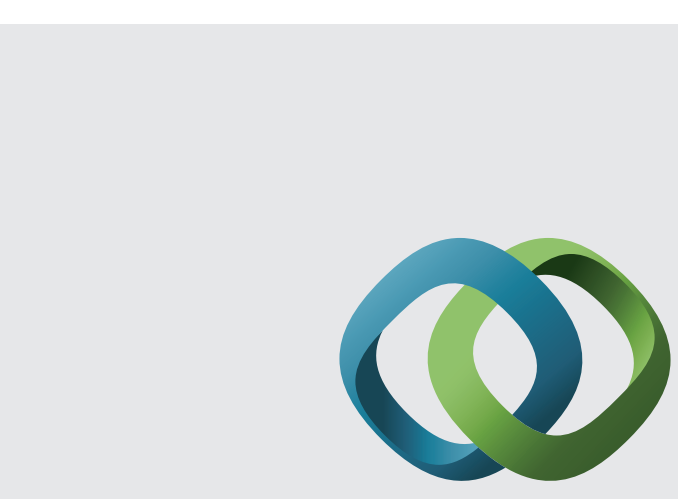

\section{Hindawi}

Submit your manuscripts at

http://www.hindawi.com
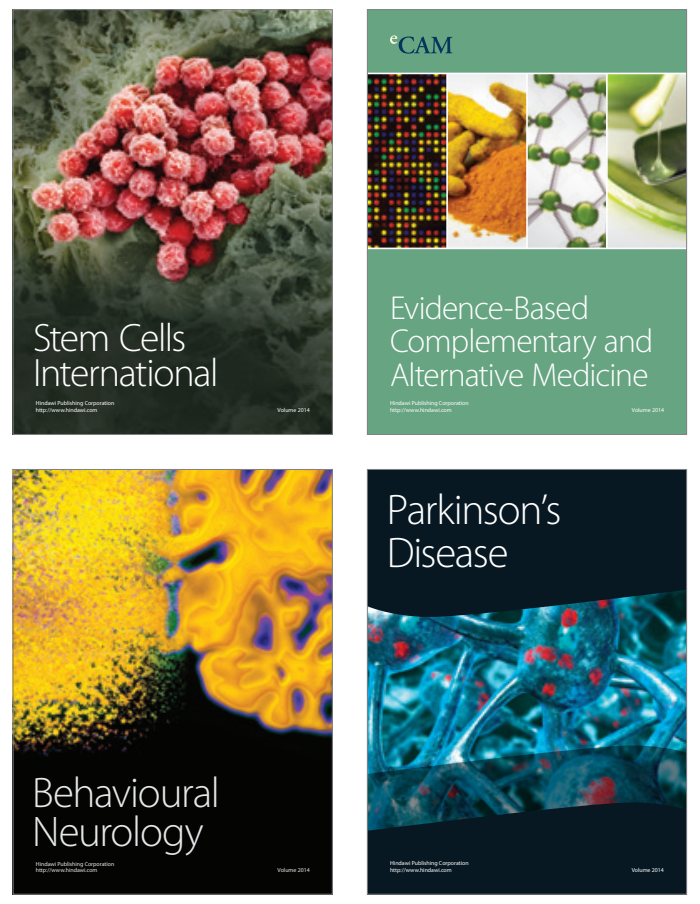
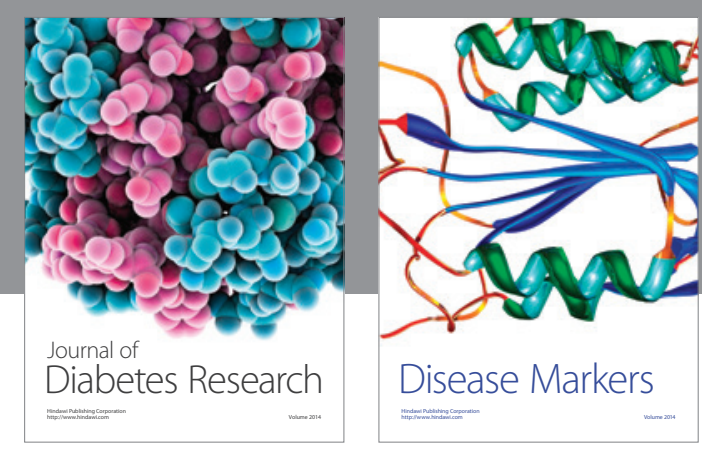

Disease Markers
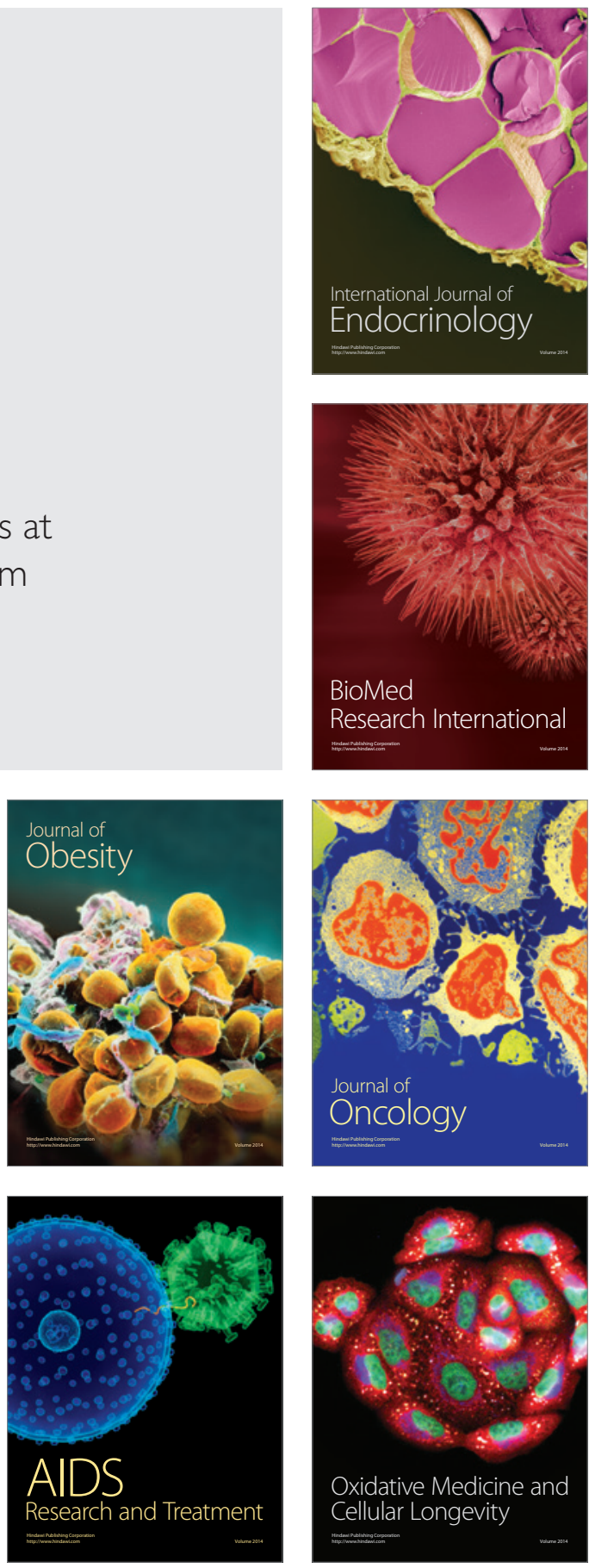